\title{
No incremental benefit of multisite atrial pacing compared with right atrial pacing in patients with drug refractory paroxysmal atrial fibrillation
}

\author{
T Levy, S Walker, S Rex, J Rochelle, V Paul
}

\begin{abstract}
Objective-To evaluate the incremental antifibrillatory effect of multisite atrial pacing compared with right atrial pacing in patients with drug refractory paroxysmal atrial fibrillation paced for arrhythmia prevention alone.

Methods-In 20 of these patients (mean (SD) age 64 (8) years; 14 female, six male), a single blinded randomised crossover study was performed to investigate the incremental benefit of one month of multisite atrial pacing compared with one month of right atrial pacing. Outcomes included the number of episodes of paroxysmal atrial fibrillation, their total duration obtained from pacemaker Holter memory, and quality of life using a cardiac specific questionnaire (the modified Karolinska questionnaire).

Results-Comparing right atrial with multisite atrial pacing, there was no significant change in either the number of paroxysmal atrial fibrillation episodes (mean (SD): right atrial pacing 77 (98) episodes $v$ multisite pacing 52 (78) episodes, NS) or their total duration (right atrial, 4.8 (5.4) days $v$ multisite, 6.3 (9.8) days, NS). Quality of life scores compared with baseline status were equally improved by either pacing strategy (mean percentage improvement: right atrial, $38 \%, \mathrm{p}=0.003$; multisite, $44 \%, \mathrm{p}=0.003$ ). There was no significant difference in life scores comparing the two pacing modes.

Conclusions-Multisite atrial pacing has no incremental antiarrhythmic effect compared with right atrial pacing in patients paced for drug refractory paroxysmal atrial fibrillation. Quality of life is equally improved with either pacing strategy, with no differences between them.

(Heart 2001;85:48-52)
\end{abstract}

Keywords: multisite atrial pacing; atrial fibrillation; pacing

Multisite atrial pacing is the technique of simultaneous activation of both the high right atrium and the inferior right atrium at the coronary sinus ostium. It has been reported that this pacing technique may improve symptoms of atrial fibrillation in patients requiring pacemaker implantation for conventional indications, when compared with either their baseline status or with a period of lone right atrial or coronary sinus ostial pacing. Furthermore it has been suggested that multisite atrial pacing may allow the discontinuation of warfarin in some patients. ${ }^{12}$

However, multisite pacing has not been assessed or compared with right atrial pacing in patients undergoing pacemaker implantation for arrhythmia prevention alone. Furthermore no assessment of this pacing technique on either quality of life or its effect on suppressing asymptomatic paroxysmal atrial fibrillation episodes has been made. The accurate detection of paroxysmal atrial fibrillation is important, as studies have suggested that $50 \%$ of episodes may be asymptomatic in fibrillating patients and yet still cause embolic events. ${ }^{3}$ Therefore continual Holter monitoring (through a pacemaker) is required for a reliable record of the presence or absence of paroxysmal atrial fibrillation.

To investigate these issues further we have performed a study in a group of patients with drug refractory paroxysmal atrial fibrillation. Our aim was to assess the incremental benefit of multisite atrial pacing compared with right atrial pacing by changes in quality of life and arrhythmia frequency. Monitoring for paroxysmal atrial fibrillation was through continual monitoring of intracardiac signals by the pacemaker.

\section{Methods}

PATIENT POPULATION

Twenty patients (mean (SD) age 64 (8) years; 14 female, six male) were given a multisite atrial pacemaker for symptomatic paroxysmal atrial fibrillation that had failed to respond to drug treatment. Pacemaker implantation was for arrhythmia prevention alone in all cases; no patient had other indications for pacing. The patients had at least three episodes of symptomatic paroxysmal atrial fibrillation a week, and had been symptomatic for 7.8 (6) years (mean (SD)). A mean of 2.8 (1.6) antiarrhythmic drugs (amiodarone in 14) had failed to control their symptoms. Antiarrhythmic and atrioventricular nodal modifying drugs remained unchanged during the follow up period and for the month (three months for amiodarone) before pacemaker implantation. The local ethics committee approved the study and all patients gave informed written consent.

The aetiology of the paroxysmal atrial fibrillation was ischaemic heart disease $(n=3)$, dilated cardiomyopathy $(\mathrm{n}=1)$, hypertension $(n=2)$, and lone $(n=14)$. Left ventricular dimensions from $M$ mode and cross sectional echocardiographic imaging were: systolic diameter, 35 (9) mm; diastolic diameter, 50 (9) $\mathrm{mm}$; and fractional shortening, 32 (6)\%. Left 
atrial diameter on $M$ mode echo was 36 (4.5) $\mathrm{mm}$ (range $27-43 \mathrm{~mm}$ ).

All patients had a DDDR pacemaker implanted (Chorum, ELA Medical, Paris, France). Leads were placed in the high right atrium (right atrial appendage), right ventricular apex, and at the coronary sinus ostium using an active fixation lead. Both atrial leads were connected through a Y adapter to the atrial port of the pacemaker, with the coronary sinus lead acting as the anode and the right atrial lead as the cathode. In this way unipolar atrial pacing provides right atrial pacing only, whereas bipolar pacing causes simultaneous biatrial pacing.

The pacemaker was programmed to DDDR with a base rate 70 beats/min and upper rate of $85 \%$ of target heart rate $(220-$ age). The mode switch was activated. Atrial sensing was programmed to maximum sensitivity $(0.4 \mathrm{mv})$ to facilitate detection of paroxysmal atrial fibrillation episodes unless there was inappropriate ventricular sensing at this level. Far field sensing was tested for by examining the atrial intracardiac and marker channels (atrial and ventricular markers both shown) for two minutes in the supine and erect position for each patient for the programmed atrial sensitivity. The postventricular atrial blanking period was left at the default setting of $162 \mathrm{~ms}$. At the end of each period, satisfactory atrial sensing and pacing was confirmed. The atrioventricular delay was programmed to allow intrinsic ventricular conduction.

Continual multisite atrial pacing was achieved by the use of an atrial resynchronised algorithm - a triggered algorithm. This operates as follows: if the patient's own intrinsic heart rhythm was slower than the pacemaker's sensor rate, both atrial sites would be paced simultaneously; however, when the patient's own heart rate was faster than that of the pacemaker sensor, the resynchronised algorithm would ensure multisite atrial pacing. This works by sensing from either lead and causing near immediate pacing ( $3 \mathrm{~ms}$ delay) of both leads when any atrial activity is detected. In practice the site where atrial activity is sensed first leads to triggered pacing into the refractory period at this site but to earlier activation at the other atrial site compared with normal intrinsic conduction.

PROTOCOL

Patients were randomised to either right atrial or multisite atrial pacing for one month and then crossed over to the other pacing mode for a further month. Patients were blinded to pacemaker programming.

MONITORING FOR ATRIAL FIBRILLATION

After each one month period the pacemaker Holter monitors were interrogated and then reset. This gave information on the number of separate episodes of paroxysmal atrial fibrillation (limited to a maximum of 510), the total duration of all these episodes (time pacemaker in fallback mode), and the stored intracardiac signal of the last eight episodes, allowing confirmation of paroxysmal atrial fibrillation.
Percentage time of atrial pacing while in sinus rhythm was also recorded.

Mode switching occurs in response to non-physiological atrial acceleration. The Chorum device is able to differentiate between normal sinus activity and premature atrial complexes. This is achieved by the use of a WARAD (window of atrial rhythm acceleration detection) algorithm. The WARAD is a period starting with an atrial event, which lasts for $62.5 \%$ of the preceding sinus interval for slow sinus rates ( $<80$ beats $/ \mathrm{min}$ ), and for $75 \%$ of the sinus interval for higher sinus rates. Normal sinus activity is defined as an event occurring outside the WARAD. A premature atrial complex is an atrial event detected within the WARAD. On sensing a premature atrial complex, the Chorum pacemaker recycles the atrial escape interval to $500 \mathrm{~ms}$ from the premature atrial complex. If no further atrial events are detected by the end of the atrial escape interval the atrium is paced with an atrioventricular delay of $109 \mathrm{~ms}$. However, if an atrial event is detected in the atrial escape interval, the pacemaker switches to a phase of suspected atrial arrhythmia. A short atrioventricular delay of $31 \mathrm{~ms}$ is initiated, with ventricular tracking limited to 120 beats/min (or lower if upper rate is lower). The WARAD is fixed during the suspicion phase and recycled after every atrial activity. The pacemaker counts for 32 cycles for arrhythmia confirmation. Atrial arrhythmia is confirmed if either 28 of the last 32 atrial cycles (good sensing of the arrhythmia), or if 18 of the last two groups of 32 cycles (poor atrial sensing) fall within the WARAD. Upon confirmation of atrial arrhythmia, the device mode switches to VDI.

QUALITY OF LIFE

This was assessed with a cardiac specific questionnaire that has been validated for pacemaker patients ${ }^{4}$ - the modified Karolinska questionnaire. Patients were asked to grade their response between 0 (no symptoms) and 10 (severe symptoms) to a series of questions on: chest pain (total score 0-40), palpitations (0-30), dizziness (0-20), shortness of breath $(0-30)$, general health $(0-10)$, and restrictions to activity $(0-10)$. This gives a total score between 0 (asymptomatic) and 140 (very symptomatic).

\section{PRIMARY END POINTS}

This was a comparison between the two pacing modes to determine if multisite atrial pacing has any incremental antifibrillatory action compared with right atrial pacing. Outcomes included the number of separate paroxysmal atrial fibrillation episodes, the total duration of all paroxysmal atrial fibrillation episodes, and the atrial fibrillation burden expressed as the percentage of total time spent in atrial fibrillation during the one month period. The percentage of time during each period when the atria were paced was also compared.

Quality of life scores were used to compare the two pacing modes with each other and with baseline status. 
Table 1 Results on the frequency of paroxysmal atrial fibrillation (PAF) comparing right atrial with multisite atrial pacing

\begin{tabular}{llll}
\hline & Right atrial & Multisite & p Value \\
\hline Number of PAF episodes & $77(98)$ & $52(78)$ & NS \\
Duration of PAF (days) & $4.8(5.4)$ & $6.3(9.8)$ & NS \\
Percentage AF burden & $14(16)$ & $19(30)$ & NS \\
Percentage of atria paced & $85(17)$ & $87(19)$ & NS \\
\hline
\end{tabular}

Values are mean (SD)

AF burden, percentage of total time spent in atrial fibrillation during the one month period.

\section{STATISTICAL ANALYSIS}

Values are given as mean (SD). Comparison between the two pacing modes for all results was with Wilcoxon's non-parametric test for two related samples.

\section{Results}

ATRIAL FIBRILLATION

These results are given in table 1. All 20 patients completed both pacing modes. There was no significant difference comparing right atrial with multisite atrial pacing in either the number of paroxysmal atrial fibrillation episodes (right atrial, 77 (98) episodes $v$ multisite, 52 (78) episodes, NS), their total duration (right atrial, 4.8 (5.4) days $v$ multisite, 6.3 (9.8) days, NS), the atrial fibrillation burden (right atrial, $14(16) \% v$ multisite, 19 (30)\%, NS), or the percentage time of atrial pacing (right atrial, 85 (17)\% v multisite, 87 (19)\%, NS).

Two patients had no paroxysmal atrial fibrillation recorded during either pacing mode. The remaining patients had further episodes during both pacing modes.

\section{QUALITY OF LIFE}

Compared with baseline status there was a significant improvement in the total Karolinska questionnaire score with both pacing modes (right atrial pacing, 38\% improvement, $\mathrm{p}=0.003$; multisite pacing, $44 \%$ improvement; $\mathrm{p}=0.003$ ) (table 2). Each individual symptom score improved with both pacing modes compared with baseline, except for general health which did not change with either mode (right atrial pacing, $8 \%$ improvement; NS; multisite pacing, $18 \%$ improvement; NS). Percentage improvements were: chest pain: right atrial pacing $44 \%, \mathrm{p}=0.01$; multisite pacing, $40 \%, \mathrm{p}=0.05$; palpitations: right atrial pacing, $43 \% ; \mathrm{p}=0.001$; multisite pacing, $52 \%$; $\mathrm{p}=0.002 ;$ dizziness: right atrial pacing, $47 \%$; $\mathrm{p}=0.05 ;$ multisite pacing, $53 \% ; \mathrm{p}=0.01$; shortness of breath: right atrial pacing, 33\%; $\mathrm{p}=0.05 ;$ multisite pacing, $41 \% ; \mathrm{p}=0.01$; restriction of activity: right atrial pacing, 33\%; $\mathrm{p}=0.01 ;$ multisite pacing, $37 \% ; \mathrm{p}=0.02$.
Comparing right atrial with multisite atrial pacing, there was no significant difference in either the total Karolinska questionnaire scores or any of the individual symptom scores.

\section{Discussion}

We have shown that in patients with drug refractory paroxysmal atrial fibrillation, paced for arrhythmia prevention alone, multisite atrial pacing has no incremental antifibrillatory effect compared with right atrial pacing. We also showed that both pacing strategies led to an improvement in quality of life compared with baseline status, without any difference between the two pacing modes. All individual symptoms were improved except for general health, which was only moderately impaired at baseline, making any significant change difficult to demonstrate.

The initial report on multisite atrial pacing compared this mode with right atrial pacing in 16 patients with drug refractory atrial fibrillation who had traditional indications for pacemaker implantation. ${ }^{2}$ Subsequently the same group reported an extension of this study to 30 patients, comparing multisite pacing with both lone right atrial and lone coronary sinus ostium pacing. ${ }^{1}$ They showed that all pacing strategies resulted in a significant increase in the symptom-free period before the recurrence of atrial fibrillation when compared with baseline status before pacemaker implantation. Multisite pacing was shown to have an additional antiarrhythmic effect over the other two pacing strategies. These results are consistent with our findings in showing that any type of pacing improves symptoms. However, we differ in that we could not show any advantage for multisite over right atrial pacing.

There were several differences in the studies that may explain these findings. First, recurrence of atrial fibrillation in the previous studies was based on symptomatic episodes confirmed by ECGs performed at routine follow up visits, 24 hour ambulatory monitoring, or transtelephonic event monitoring. No data are available on long term pacemaker Holter analysis. They may therefore have underestimated the true incidence of atrial fibrillation. This is supported by studies in patients paced for bradycardic indications with associated paroxysmal atrial fibrillation which report that $50 \%$ of episodes of paroxysmal atrial fibrillation recorded on pacemaker Holters can be asymptomatic. ${ }^{3}$ Therefore continual monitoring through pacemaker Holters for paroxysmal atrial fibrillation is necessary, especially if

Table 2 Results for the Karolinska questionnaire score (KQ) and individual symptom scores at baseline, after one month of right atrial pacing, and after one month of multisite atrial pacing

\begin{tabular}{lllllll}
\hline & Baseline (B) & Right atrial (RA) & $\begin{array}{l}\text { p Value, } \\
R A v B\end{array}$ & Multisite (M) & $\begin{array}{l}p \text { Value, } \\
M v B\end{array}$ & $\begin{array}{l}p \text { Value, } \\
M v R A\end{array}$ \\
\hline Total KQ score (0-140) & $55(28)$ & $34(31)(-38 \%)$ & 0.003 & $31(27)(-44 \%)$ & 0.003 & NS \\
Chest pain (0-40) & $9.7(9.8)$ & $5.4(7.8)(-44 \%)$ & 0.01 & $5.8(7.4)(-40 \%)$ & 0.05 & NS \\
Palpitations (0-30) & $17.8(7.7)$ & $10.2(8.0)(-43 \%)$ & 0.001 & $8.6(6.8)(-52 \%)$ & 0.002 & NS \\
Dizziness (0-20) & $8.1(6.6)$ & $4.3(6.2)(-47 \%)$ & 0.05 & $3.8(4.5)(-53 \%)$ & 0.01 & NS \\
SOB (0-30) & $10.3(7.8)$ & $6.9(7.5)(-33 \%)$ & 0.05 & $6.1(6.5)(-41 \%)$ & 0.01 & NS \\
Restrictions (0-10) & $5.1(2.1)$ & $3.4(3.2)(-33 \%)$ & 0.01 & $3.2(3.3)(-37 \%)$ & 0.02 & NS \\
General health (0-10) & $3.7(2.7)$ & $3.4(3.0)(-8 \%)$ & NS & $3.0(3.0)(-18 \%)$ & NS & NS
\end{tabular}

Values are mean (SD), with mean percentage change from baseline shown in brackets.

SOB, shortness of breath. 
advice on withdrawal of anticoagulation is required.

A further limitation in these former studies included a run in period following pacemaker implantation. This was to allow for antiarrhythmic drug optimisation before monitoring for symptomatic arrhythmic episodes began. This therefore invalidates any comparison with baseline arrhythmia frequency, as drug treatment had been altered. In our study all drugs remained unchanged. Furthermore their mechanism of multisite pacing was dependent on atrial overdrive to ensure capture at both atrial sites. The percentage of atrial pacing was not reported; however, it is unlikely that this would approach $100 \%$, and so multisite activation was not achieved continuously. The effect of this would to underestimate the effectiveness of multisite pacing. In our study a triggered algorithm was used to ensure multisite pacing at all heart rates.

Other differences include the design of the studies. Our patients underwent a randomised crossover study comparing the pacing modes, while the previous studies were consecutive designs with a period of multisite pacing proceeding right atrial pacing. This could bias one of the pacing modes. Our follow up was limited to one month in each pacing mode, compared with three months and six months in the previous studies. This would tend to weaken our study. However, despite this shorter time period $90 \%$ of our patients had arrhythmia reccurrence with both pacing modes, compared with $38 \%$ with right atrial and $11 \%$ with multisite pacing in the previous studies.

Finally the populations studied were totally different and this may explain the disparate findings. In Saksena's study ${ }^{2}$ the patients had conventional indications for pacemaker implantation. The majority $(73 \%)$ also had underlying organic heart disease, with several different types of atrial arrhythmia including paroxysmal and persistent atrial fibrillation, atrial flutter, and atrial tachycardia. Our patients were paced for arrhythmia prevention alone, the majority $(70 \%)$ had no organic heart disease, and all had paroxysmal atrial fibrillation as their only atrial arrhythmia. In our patients with organic heart disease $(n=6)$ there was still no additional benefit from multisite pacing. Therefore multisite atrial pacing may only have incremental benefits over right atrial pacing in patients with organic heart disease with conventional indications for pacing.

Short term electrophysiological studies using temporary pacing leads placed either within the coronary sinus or at the ostium, combined with a high right atrial lead, have shown that multisite pacing can suppress the induction of atrial fibrillation caused by high right atrial extrastimuli in $55-66 \%$ of patients. ${ }^{56}$ In these patients with suppression, an increase in dispersion of refractoriness between the two pacing sites is found at baseline, and multisite pacing can decrease conduction delay to all areas of the atria. In the non-suppressible patients, however, there is no change in conduction times following a high right atrial extrastimulus and less dispersion of refractoriness. Furthermore, studies assessing the onset of paroxysmal atrial fibrillation using pacemaker Holters and conventional 24 hour Holter monitors have shown several different onset mechanisms between and within patients. ${ }^{78}$ Only approximately $50 \%$ of onset episodes were preceded by atrial ectopic beats, where it may be anticipated that multisite pacing would have an antiarrhythmic effect.

Therefore acute electrophysiological studies suggest there may be responders and nonresponders to multisite pacing, while Holter monitoring suggests that multisite pacing would need to prevent several different onset mechanisms. Future studies are required to determine whether acute findings of atrial fibrillation suppression could predict long term success with multisite atrial pacing and thus identify a group that may get benefit from this pacing strategy. In our study there was a subgroup of eight patients $(40 \%)$ who appeared to gain additional benefit from multisite pacing compared with right atrial pacing, with a significant reduction in their total duration of paroxysmal atrial fibrillation (right atrial pacing, 5.6 (4.6) days $v$ multisite pacing, 1.5 (1.5) days; $p=0.01)$. No baseline clinical feature could identify these patients. However, these results may have been the result of random chance and the effect of selecting a few responding patients from the total population.

\section{STUDY LIMITATIONS}

Our arrhythmia frequency was dependent on the accurate function of the pacemaker Holter, which has been validated elsewhere. ${ }^{9}$ Stored intracardiac signals allowed confirmation of the arrhythmia, and each patient acted as their own control without any alteration in atrial sensing. It is impossible to exclude some false mode switching owing to far field sensing or noise; however, this should affect both pacing modes equally.

Our follow up period was limited to a relatively short period of one month in each mode. However, within the initial one month period there were on average 77 separate episodes of paroxysmal atrial fibrillation lasting a mean duration of over five days. This frequency gives an adequate period for data collection for statistical analysis. Furthermore, from previous experience investigating the role of biatrial pacing with three month randomisation periods in a separate population, we found that many patients $(25 \%)$ requested atrioventricular ablation during the follow up period because of continued symptoms. ${ }^{10}$ In biatrial pacing the second atrial lead is placed within the coronary sinus to pace the left atrium directly, thus producing biatrial (right and left) pacing. This is different from the multisite atrial pacing investigated in this study, where the second atrial lead is placed at the coronary sinus ostium, producing dual site right atrial pacing. As there is the suggestion that arrhythmia frequency may increase following atrioventricular ablation, ${ }^{11-13}$ we wanted to avoid this confounding factor so we limited the follow up to two months. No patient underwent ablation during our study. 
CONCLUSION

Multisite atrial pacing has no incremental antiarrhythmic effect compared with right atrial pacing in patients paced for drug refractory paroxysmal atrial fibrillation. Quality of life is equally improved compared with baseline with either pacing strategy, but with no further benefit from multisite pacing.

1 Delfaut P, Saksena S, Prakash A, et al. Long-term outcome of patients with drug refractory atrial flutter and fibrillation cing for arrhythmia prevention. F Am Coll Cardiol 1998;32:1900-8

prevention. F Am Coll Cardiol 1998;32:1900-8. fibrillation with chronic dual site right atrial pacing. $\mathcal{f} \mathrm{Am}$ Coll Cardiol 1996;28:687-94.

3 Garrigue S, Barold S, Cazeau S, et al. Prevention of atrial arrhythmias during DDD pacing by atrial overdrive. PACE arrhythmias durin

4 Linde C. How to evaluate quality of life in pacemaker patients: problems and pitfalls. PACE 1996;19:391-7.

5 Yu WC, Chen SA, Tai CT, et al. Effects of different atria pacing modes on atrial electrophysiology. Implicating the mechanism of biatrial pacing in the prevention of atria fibrillation. Circulation 1997;96:2992-6.
6 Prakash A, Saksena S, Hill M, et al. Acute effects of dual-site right atrial pacing in patients with spontaneous and inducright atrial pacing in patients with spontaneous and induc1997; 29:1007-14

7 Janko S, Dorwarth U, Gerth C, et al. Analysis of onset scenarios in patients with drug-refractory paroxysmal atrial fibrillation: implications for preventive pacing strategies [abstract]. PACE 1999;22:A172.

8 Hnatkova K, Waktare JEP, Murgatroyd FD, et al. Analysis of the cardiac rhythm preceding episodes of paroxysmal atrial fibrillation. Am Heart $\mathcal{F}$ 1998;135:1010-19.

9 Defaye P, Dournaux F, Mouton E, for the AIDA multicentre study group. Prevalence of supraventricular arrhythmia from the automated analysis of data stored in the DDD pacemakers of 617 patients: the AIDA study. PACE 1998;21:250-5.

10 Levy T, Walker S, Rochelle J, et al. Evaluation of bi-atrial pacing, right atrial pacing and no pacing, in patients with drug refractory atrial fibrillation. Am f Cardiol 1999;84:426-9.

11 Mitchell MA, Ackerman SJ, Nath S, et al. Atrial rhythm after atrioventricular junctional ablation. Am $f$ Cardiol 1996;78:1251-4

12 Gribbin GM, Bourke JP, McComb JM. Predictors of atrial rhythm after atrioventricular node ablation for the treatment of paroxysmal atrial arrhythmias. Heart 1998;79:548-53.

13 Gianfranchi L, Brignole M, Menozzi C, et al. Progression of permanent atrial fibrillation after atrioventricular junction ablation and dual-chamber pacemaker implantation in patients with paroxysmal atrial tachyarrhythmias. $A m \mathcal{F}$ Cardiol 1998;81:351-4.

\section{IMAGES IN CARDIOLOGY}

\section{Cardiac arrest caused by spontaneous left coronary artery dissection in a young woman}

A 39 year old woman was admitted on December 1999 after resuscitation from cardiac arrest, which occurred during Holter monitoring (ST segment elevation followed by ventricular fibrillation was documented). The woman complained of recent onset chest discomfort with syncope. She had no coronary risk factors, no drug abuse, and no significant diseases in her history, although she suffered from migraine (which was being treated with sumatriptan $50 \mathrm{mg} /$ day). On arrival, the patient had pronounced anterolateral ST segment elevation; infusion of nitrates, heparin, and $\beta$ blockers provided prompt clinical evidence of reperfusion. For this reason, coronary arteriography was delayed.

Four days later, the patient had recurrent chest pain at rest, with unexpected ST segment elevation in inferior leads, and hypotension $(80 / 45 \mathrm{~mm} \mathrm{Hg})$. Urgent coronary arteriography was carried out.

Angiographic findings are shown $\left(15^{\circ}\right.$ right anterior oblique, $10^{\circ}$ cranial view). A long dissection involved the whole left main coronary stem (LM), with contrast dye persisting outside the lumen, and extended both to the left anterior descending artery (LAD) and to the left circumflex artery (LCX). In the LAD, persistence of extraluminal contrast was evident in the proximal segment, while a radiolucent linear streak could be noted in the whole mid portion; in the LCX the dissection involved the proximal segment and ended in a tight stenosis of the true lumen, with impaired anterograde flow.

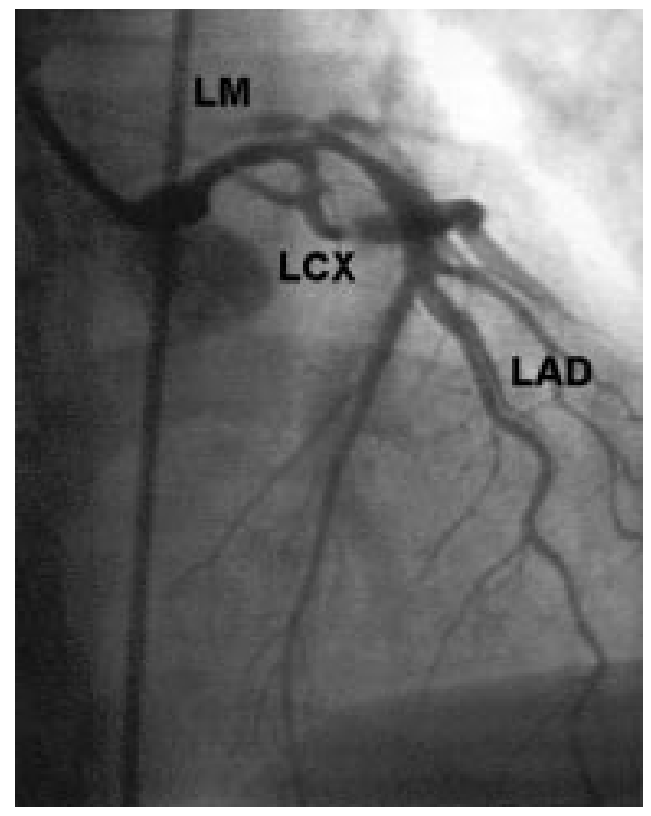

Intra-aortic balloon pumping was immediately started, followed by haemodynamic improvement. A double coronary artery bypass graft was successfully performed the same day (left internal mammary artery to LAD, saphenous vein graft to obtuse marginal branch).

D TAVELLA M TURRI

P ZARDINI 